Journal of Engineering and Applied Sciences 14 (Special Issue 5): 9009-9013, 2019

ISSN: 1816-949X

(C) Medwell Journals, 2019

\title{
Smart Hypertension Care Architecture Development based on the Use Case and Reasoning Method
}

\author{
${ }^{1}$ Kyunghan Chun, ${ }^{2}$ Yongjun Jon and ${ }^{3}$ Jiwon Yang \\ ${ }^{1}$ School of Electronic and Electrical Engineering, Daegu Catholic University, \\ 38430 Gyeongbuk, Korea \\ ${ }^{2}$ Department of Computer Science and Engineering, Kyungpook National University, \\ 41566 Daegu, Korea \\ ${ }^{3}$ Department of Nursing, Kyungil University, 38428 Gyeongbuk, Korea
}

\begin{abstract}
High Blood Pressure (BP, hypertension) is a leading chronic condition and has become the main risk factor for many high-risk diseases such as heart attacks. But hypertension is a chronic disease which can be effectively prevented and controlled only, if the physiological parameters of the patient are constantly monitored, along with the full support of the health education and professional medical care. To achieve the early diagnosis of hypertension and smart healthcare a smart healthcare architecture has been designed. Smart mobile healthcare system has been designed to improve patient-doctor interaction and to promote the self-management of chronic diseases by the patients themselves. The developed system provides intelligent, user-friendly and secure, medical and well-being decision support through embedded software in mobile devices by utilizing specific sensors and data from customized information systems. In this study, a reasoning method for smart healthcare is proposed for hypertension management and all systems are integrated and tested for evaluations.
\end{abstract}

Key words: Sliding mode control, compensator, disturbance, hypertension, medical care, healthcar

\section{INTRODUCTION}

In recent years, healthcare for elderly people has been an important research topic. The commonly seen chronic diseases for elderly people include hypertension and arrhythmia. The current healthcare for such diseases is still mainly from outpatient services. Due to the development of Information and Communication Technology (ICT) the feasibility of home telecare has been highly raised.

It is important to integrate wireless communication technology for modern healthcare systems (Lin et al., 2004; Anliker et al., 2004; Rasid and Woodward 2005; Kafeza et al., 2004; Lee et al., 2005, 2006; Guey et al., (2006) have utilized a Personal Digital Assistant (PDA) to monitor and collect the physiological parameters extracted by a physiological signal module attached to patients. Home telecare service has been further extended to become mobile care service (Anliker et al., 2004; Rasid and Woodward 2005; Kafeza et al., 2004; Lee et al., 2005; Guey et al., 2006) due to the ubiquity of Global System for Mobile communications (GSM) and General Packet Radio Service (GPRS) and broadly applied to the design of a healthcare system to utilize a GSM/GPRS mobile network to provide healthcare service with functions of emergency alerts and early warning messages (Anliker et al., 2004; Kafeza et al., 2004; Lee et al., 2006; Guey et al., 2005, 2006).

Blood Pressure (BP) monitoring as practiced in the developing world is an expensive proposition. High BP becomes known to patients when they are confronted with serious diseases such as atherosclerotic cardiovascular disease which may result from hypertension. There are several challenges; for instance, lack of cheap intelligent home-care BP measurement devices or having to travel long distances for medical treatment because of limited physical infrastructure or an unbalanced distribution of medical resources. The smart mobile healthcare system has been developed where smart refers to the ability of drawing clinical conclusions about the patient's health status without or with limited, human intervention.

This study accomplishes the evaluation and verification of the product developed for hypertension use case from both the system and the user perspective. Although, the main focus of this study is the user perspective, this strategy gives us a valuable insight into whether the design of the system has been appropriate for the real world.

Corresponding Author: Jiwon Yang, Department of Nursing, Kyungil University, Gyeongbuk 38428, Korea, ykyee@kiu.kr 


\section{MATERIALS AND METHODS}

Smart healthcare architecture: The general architecture of the smart healthcare system consists of devices that collect information directly from the user or from databases that contain user data, typically in electronic health records. The essence of an mHealth architecture is that the user obtains appropriate feedback from the mobile device through the interpretation of available data. Such feedback is provided by an embedded decision-support system. The architecture of the smart healthcare system is shown in Fig. 1 which is composed of front-end (right) and back-end (left). The front-end has mobile devices, medical sensors, environmental sensors, etc. and the back-end has a server and a database. Using this architecture, each user case is developed along the functions of sensing, reasoning, alarm and recommendation, etc.
Hypertension care with reasoning method: The hypertension use cas consists of medical sensors, software and computer systems as shown in Fig. 2. Medical devices have been certified by related organization, so, testing environment focus on front-end, hypertension apps and back-end server.

The hypertension use case identified the actors involved in an interaction with the systems and sensor. The major actors are patients a doctor and an independent test engineer. A patient measures his hypertension according to a registered schedule and a test engineer checks the result using patient's smartphone. A test engineer validates the hypertension app and server functionalities with the requirements and specification and registers test results on an Excel sheet. A test engineer also, can be patient, if he/she has hypertension.

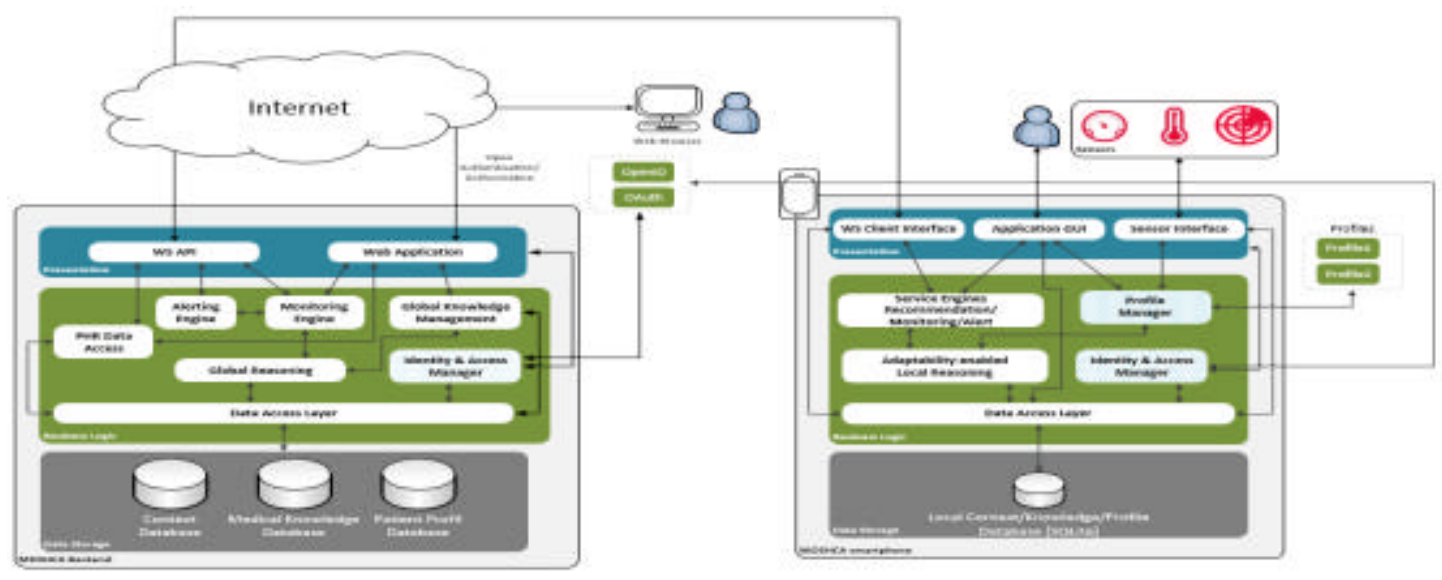

Fig. 1: Smart healthcare architecture

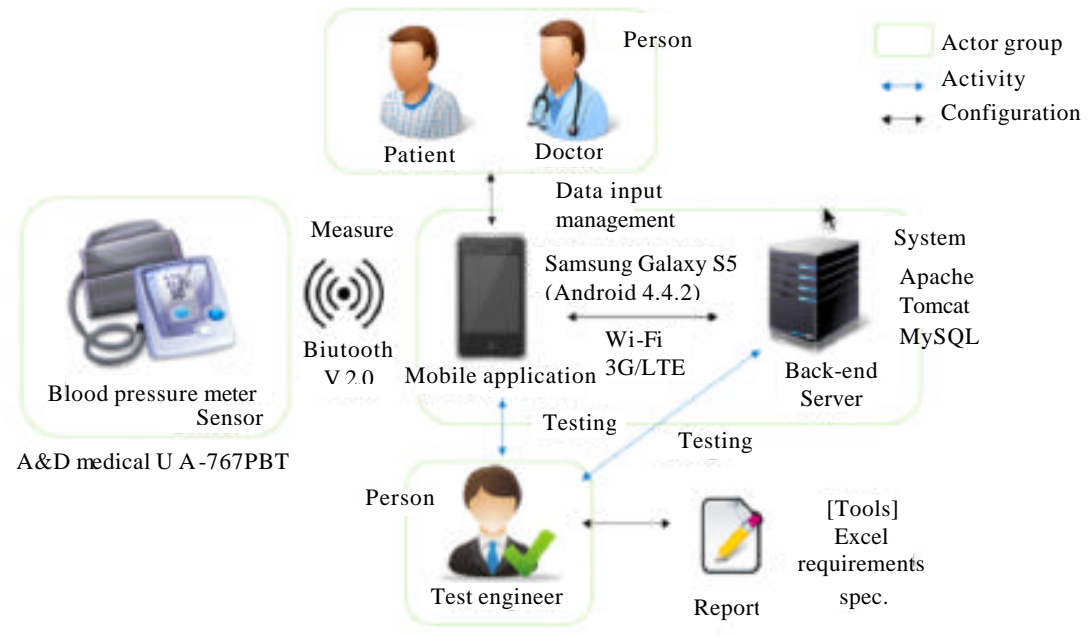

Fig. 2: Hypertension use case 


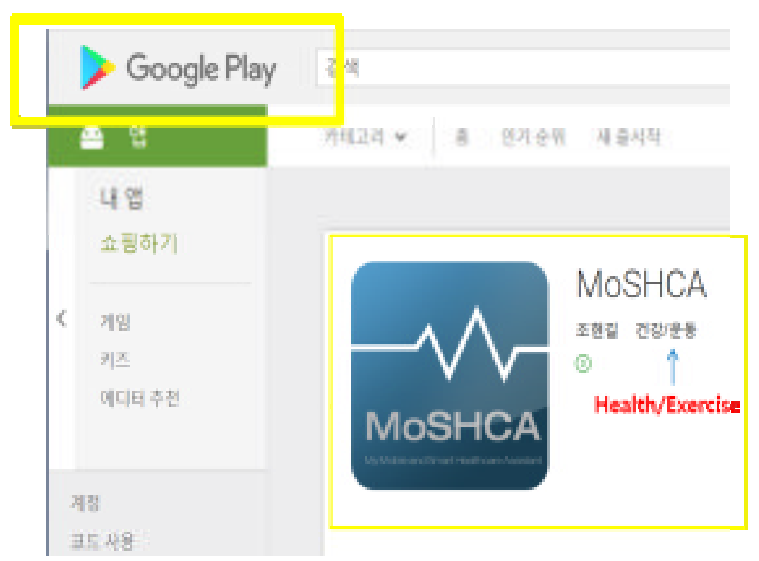

Fig. 3: Hypertension app on Google play

Hypertension is the most common disease for seniors. Approximately, $40 \%$ of the global senior population on earth has hypertension. Also, hypertension is the most common reason for visiting the hospital. The hypertension mobile application monitors blood pressure patients with blood pressure meter by him self/herself. The application supports major blood pressure meters that implemented the IEEE 11073 Standard. Also, hypertension application supports physical activity and medication to increase adherence for hypertension management.

The hypertension mobile application was uploaded on the Google Play App store as shown in Fig. 3. For the hypertension use case a blood pressure meter is used to acquire Systolic and Diastolic Blood Pressure (SBP, DBP). Optionally a customized activity sensor could be used to receive patient exercise related data. A blood pressure meter specification is as follows:

- Measurement range: $20-280 \mathrm{mmHg}$, pulse $40-200 \mathrm{bpm}$

- Accuracy: $+/-2 \%$ of blood pressure

- Method: oscillometric

- Communication protocols to be supported: IEEE 11073 standard, Bluetooth Version 2.0 and above

- Medical standards: ANSI/AAMI SP-10

- Blood pressure data memory to be stored: more than 30 measurements

- Power: must be operated using battery

The recommended blood pressure meter is $A \& D$ Medical UA-767PBT or Continua Health Alliance certified sensors in Fig. 4. The hypertension application uses rule-based reasoning mechanism to determine the cause of high blood pressure. The rule-based reasoning architecture involves two principal components as a series of static rules and applied database.

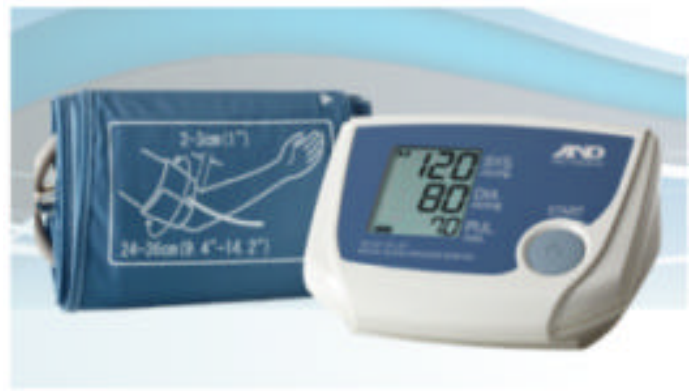

Fig. 4: A\&D medical UA-767PBT

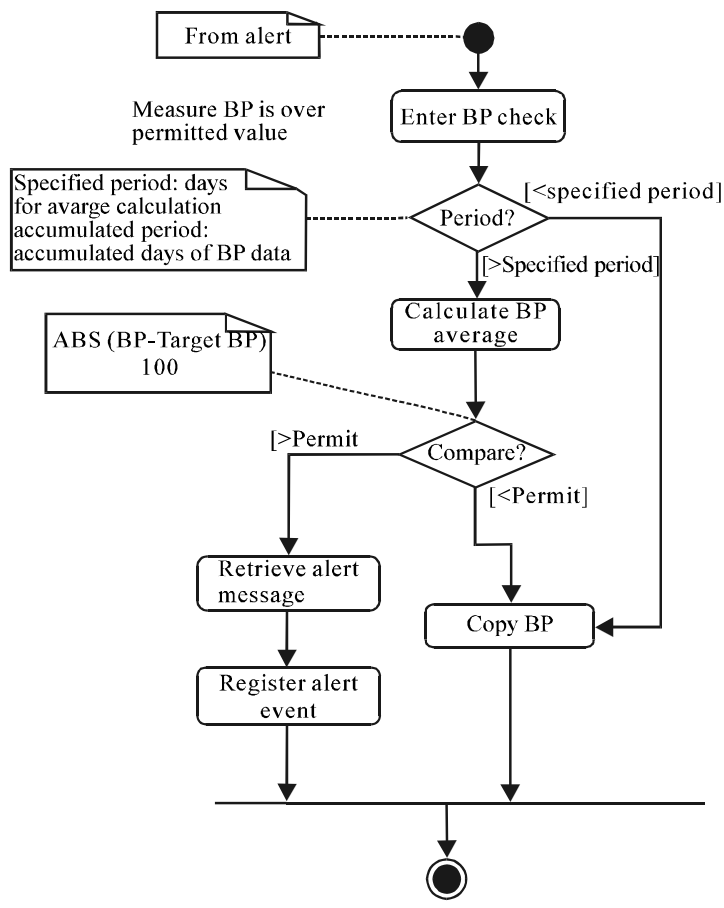

Fig. 5: Hypertension decision flow

The hypertension application determines the blood pressure state by analyzing collected blood pressure values and its related information. Figure 5 shows that how to determine hypertension decision using measured blood pressure values and parameters. In order to give the exact decision to patient, parameters are provided such as period, alert level and target value.

If an alert is registered, the hypertension application determines the action according to level of severity. The hypertension application provides four action lists in response to the evaluation of the alert as shown in Table 1.

User verification: For the verification, patients or users and carer-givers try out the technical set ups in 
Table 1: Alert level of hypertension

\begin{tabular}{lll}
\hline Levels & Conditions & Action list \\
2 & Alert level $=1$ & $\begin{array}{l}\text { Display alert on the smart phone application } \\
\text { Display alert on the smart phone application }\end{array}$ \\
3 & $\begin{array}{l}\text { Alert level }=2 \\
\text { Alert level }=3 \text { or }\end{array}$ & $\begin{array}{l}\text { Display alert on the smart phone application send notification to doctor } \\
\text { Alert level } 2>\text { continuous 2 times } \\
\text { Alert level }=4 \text { or } \\
\text { Alert level } 3>\text { continuous 2 times }\end{array}$ \\
& $\begin{array}{l}\text { Display alert on the smart phone application send notification to doctor } \\
\text { Send emergency message to registered person }\end{array}$ \\
\hline
\end{tabular}

Table 2: Questionnaire for usability test

Results

Strongly disagree/strongly agree

Questionnaire

12

I am satisfied with how easy it is to use this application

I was able to complete the blood measurement process using this application

I felt comfortable using this sy stem

The application gave notifications that clearly told me action to take

Whenever I made a mistake using an application, I could recover easily quickly

The user interface of this application was pleasant

The application has all the functions and capabilities I expected it to have

collaboration with medical and health personnel. User verification for hypertension is the test process to validate system requirements in terms of user experiences. This process is very important to build the right product that satisfies specification and user functional requirements as well as user experiences.

Goals are to manage their hypertension with smart healthcare application by collected blood pressure related data and to analyze collected data and to decide right actions for the hypertension changes at real life. Test environment is as follows:

Group target: Patients in hypertension stage level 1 and senior people in prehypertension: 3 persons.

Environment: Validation will be done at patient home and at the company research center. Also, a blood pressure meter is used to measure blood pressure and pulse.

Goals: Finding a better decision making for hypertension patient using rule-based reasoning.

Procedure: We have uploaded hypertension application for testing on the Google paly. We get comments and review from downloaded users. In order to get correct answers, we used a questionnaire table as shown in Table 2 .

\section{RESULTS AND DISCUSSION}

We have tested two users and testing results are described in Table 3. Due to limited budget allowance, we tested only 2 users. However, we collected good feedback
Table 3: Summary of user comments

\begin{tabular}{|c|c|}
\hline $\begin{array}{cc} & \text { Ages, } \\
\text { User name } & \text { hypertension level } \\
\end{array}$ & $\begin{array}{l}\text { Scores ( } 7 \text { questions, max.score: } 35 \text { ) } \\
\text { and comments }\end{array}$ \\
\hline \multicolumn{2}{|l|}{1} \\
\hline \multirow[t]{10}{*}{48 level 1 male } & Total score: 30 \\
\hline & Question 1: 5 \\
\hline & Question 2: 5 \\
\hline & Question 3: 4 \\
\hline & Question 4: 4 \\
\hline & Question 5: 3 \\
\hline & Question 6: 4 \\
\hline & Question 7: 5 \\
\hline & Comments: \\
\hline & $\begin{array}{l}\text { The pre-defined rules are not matched } \\
\text { patient's for mehy pertension state and } \\
\text { are still difficult to understand }\end{array}$ \\
\hline \multicolumn{2}{|l|}{2} \\
\hline 46 pre-hypertension & Total score: 27 \\
\hline \multirow{9}{*}{ female } & Question 1: 5 \\
\hline & Question 2: 4 \\
\hline & Question 3: 4 \\
\hline & Question 4: 4 \\
\hline & Question 5: 3 \\
\hline & Question 6: 3 \\
\hline & Question 7:4 \\
\hline & Comments: \\
\hline & $\begin{array}{l}\text { In the graphic view screen, font size } \\
\text { is small to see it clearly }\end{array}$ \\
\hline
\end{tabular}

from them. The users still feel that the pre-defined rules are difficult to use. Also, the user interface is not easy to use for the elderly. In order to reduce the difficulties of rules input, we created pre-defined rules that reflected hypertension states and its related factors. Patients just needed to select a rules according to his/her hypertension level and other factors. However, these rules are still not enough to include all the states of patients, so, they must adjust setting by themselves or by requesting help to doctor. 


\section{CONCLUSION}

In this study, the hypertension application is verified through verification and evaluation procedures. The non-functional requirements are also validated but some test cases could not be carried out because of test bed limitations. The results and discussions contained herein act as test beds for the application of smart healthcare technology. They provide important experience and feedback on how the technology works in real life settings. We are investigating how to enhance the reasoning mechanism and rules and analyzing relevant factors for a better decision procedure.

\section{ACKNOWLEDGEMENT}

This research was supported by Kyungil University. This research was also supported by the Basic Science Research Program through the National Research Foundation of Korea (NRF) funded by the Ministry of Education (No. NRF-2015R1D1A1A01059060).

\section{REFERENCES}

Anliker, U., J.A. Ward, P. Lukowicz, G. Troster and F. Dolveck et al., 2004. AMON: A wearable multiparameter medical monitoring and alert system. IEEE. Trans. Inf. Technol. Biomed., 8: 415-427.
Guey, R.L.E.E., C.C. Hsiao, C.C.H.E.N. Chung and M.L.I.U. Shiu, 2006. A mobile-care system integrated with Bluetooth blood pressure and pulse monitor and cellular phone. IEICE. Trans. Inf. Syst., 89: 1702-1711

Kafeza, E., D.K. Chiu, S.C. Cheung and M. Kafeza, 2004. Alerts in mobile healthcare applications: Requirements and pilot study. IEEE. Trans. Inf. Technol. Biomed., 8: 173-181.

Lee, R.G., C.C. Hsiao, K.C. Chen and M.H. Liu, 2005. An intelligent diabetes mobile care system with alert mechanism. Biomed. Eng. Appl. Basis Commun., 17: 186-192.

Lee, R.G., C.C. Lai, S.S. Chiang, H.S. Liu and C.C. Chen et al., 2006. Design and implementation of a mobile-care system over wireless sensor network for home healthcare applications. Proceedings of the 28th Annual International Conference on the IEEE Engineering in Medicine and Biology Society, August 30-3 September, 2006, IEEE, Taipei, Taiwan, ISBN: 1-4244-0032-5, 6004-6007.

Lin, Y.H., I.C. Jan, P.I. Ko, Y.Y. Chen and J.M. Wong et al., 2004. A wireless PDA-based physiological monitoring system for patient transport. IEEE. Trans. Inf. Technol. Biomed., 8: 439-447.

Rasid, M.F.A. and B. Woodward, 2005. Bluetooth telemedicine processor for multichannel biomedical signal transmission via mobile cellular networks. IEEE. Trans. Inf. Technol. Biomed., 9: 35-43. 